\title{
Determinants of change in physical activity during moderate-to-severe COPD exacerbation
}

\author{
This article was published in the following Dove Press journal: \\ International Journal of COPD \\ 3 February 2016 \\ Number of times this article has been viewed
}

\author{
Cristóbal Esteban ${ }^{1,2}$ \\ José M Quintana ${ }^{2,3}$ \\ Susana Garcia-Gutierrez ${ }^{2,3}$ \\ Ane Anton-Ladislao ${ }^{3}$ \\ Nerea Gonzalez ${ }^{2,3}$ \\ Marisa Baré2,4 \\ Nerea Fernández de \\ Larrea $^{2,5}$ \\ Francisco Rivas-Ruiz ${ }^{2,6}$ \\ For the IRYSS-COPD group \\ 'Respiratory Department, Hospital \\ Galdakao-Usansolo, Bizkaia; ${ }^{2}$ Red de \\ Investigación en Servicios Sanitarios y \\ Enfermedades Crónicas (REDISSEC), \\ Galdakao; ${ }^{3}$ Research Unit, Hospital \\ Galdakao-Usansolo, Bizkaia; ${ }^{4}$ Unit of \\ Clinical Epidemiology, Corporacio \\ Parc Tauli, Barcelona; ${ }^{5} \mathrm{Health}$ \\ Department, Madrid; ${ }^{6}$ Research Unit, \\ Hospital Costa del Sol, Mabella, \\ Málaga, Spain
}

\begin{abstract}
Background: Data are scarce on patient physical activity (PA) level during exacerbations of chronic obstructive pulmonary disease (eCOPD). The objective of the study was to evaluate the level and determinants of change in PA during an eCOPD.

Materials and methods: We conducted a prospective cohort study with recruitment from emergency departments (EDs) of 16 participating hospitals from June 2008 to September 2010. Data were recorded on socioeconomic characteristics, dyspnea, forced expiratory volume in 1 second (FEV1\%), comorbidities, health-related quality of life, factors related to exacerbation, and PA in a stable clinical condition and during the eCOPD episode.

Results: We evaluated 2,487 patients. Common factors related to the change in PA during hospital admission or 7 days after discharge to home from the ED were lower PA at baseline and during the first 24 hours after the index evaluation. Age, quality of life, living alone, length of hospital stay, and use of anticholinergic or systemic corticosteroids in treating the exacerbation were associated with the change in PA among hospitalized patients. Predictors of change among patients not admitted to hospital were baseline FEV1\% and dyspnea at rest on ED arrival.

Conclusion: Among the patients evaluated in an ED for an eCOPD, the level and change in PA was markedly variable. Factors associated with exacerbation (PA 24 hours after admission, medication during admission, and length of hospital stay) and variables reflecting patients' stable clinical condition (low level of PA, age, quality of life, FEV1\%) are predictors of the change in PA during a moderate-to-severe eCOPD.
\end{abstract}

Keywords: COPD exacerbations, physical activity, prospective cohort study

\section{Introduction}

Among older individuals, there is a complex association between functional decline, defined as loss of capacity to carry out activities of daily life, ${ }^{1,2}$ and hospitalization. It is well known that hospitalization can lead to functional decline. ${ }^{1,2}$ Sometimes, however, functional decline precedes hospitalization. ${ }^{3}$ The etiology of functional decline goes beyond the disease process that triggered the admission and it is likely that several mechanisms are involved. One possible contributor is decreased mobility - a reduction in an individual's usual level of physical activity (PA). During hospitalization, geriatric patients spend $83 \%$ of their time in bed and just $4 \%$ standing or walking. ${ }^{4}$ Low mobility in hospitalized patients has been shown to be a predictor of adverse outcomes. ${ }^{5}$

After adjusting for demographic and clinical characteristics, older patients who increased their walking distance by 600 steps (equivalent to walking for 12 minutes) from the first day of admission to the second day were discharged 2.13 days before those who did not achieve this level of PA. ${ }^{6}$

Relatively little is known about the association between change in PA level and exacerbation-related outcomes in patients with COPD. However, the evidence base has begun to grow and it has been seen that PA may have an influence on very
Correspondence: Cristóbal Esteban Servicio de Neumología, Hospital de Galdakao-Usansolo, Barrio Labeaga s/n, 48960 Galdakao, Bizkaia, Spain

Tel +34944007002

$\mathrm{Fax}+34944007002$

Email cristobal.est@gmail.com 
important outcomes, such as mortality and readmissions. ${ }^{7,8}$ During exacerbations, COPD patients report even less daily outdoor PA, ${ }^{9}$ and their level of PA falls even further during hospitalization for an exacerbation of COPD (eCOPD). ${ }^{10}$ In fact, patients often do not recover their PA level within the first month after an eCOPD. ${ }^{10}$

The aim of this study was to evaluate changes in PA during episodes of moderate-to-severe eCOPD requiring assessment in an emergency department (ED) and identify determinants of these changes.

\section{Methods/design}

This prospective cohort study recruited patients from 16 hospitals in the Spanish National Health Service. Patients with an eCOPD attending the ED were informed about the study objectives and invited to participate, and if they agreed, written informed consent was obtained. All data collected were kept confidential and the Institutional Review Boards of the participating hospitals approved this study. Recruitment started in June 2008 and ended in September 2010. A description of the study protocol has been published. ${ }^{11}$

Patients were eligible for the study if they arrived at the ED with symptoms consistent with an eCOPD. COPD was confirmed by spirometry if the patient had a forced expiratory volume in 1 second/forced vital capacity (FEV1/FVC) ratio $<70 \%$. The severity of airflow limitation was classified in accordance with the Global Initiative for Chronic Obstructive Lung Disease (GOLD). ${ }^{12}$

Exacerbation was defined as an event characterized by a change in the patient's baseline dyspnea, cough, and/or sputum that was beyond normal day-to-day variations and may have warranted a change in medication. ${ }^{12}$ Patients newly diagnosed with COPD in the ED were included in the study, provided that the diagnosis was confirmed by spirometry within 60 days after the index episode at a time when they were stable. Patients were excluded from the study if they did not wish to participate or if, at the time they were being evaluated in the ED, their eCOPD was complicated by a comorbidity such as pneumonia, pneumothorax, pulmonary embolism, lung cancer, or left cardiac failure.

\section{Data collection}

Data collected upon arrival at the ED included socioeconomic data, information about the patient's respiratory state (arterial blood gases, respiratory rate, dyspnea), level of consciousness measured by the Glasgow Coma Scale, ${ }^{13}$ and presence of other conditions included in the Charlson Comorbidity Index. ${ }^{14}$
For patients admitted to the hospital from the ED, we collected additional data from the patient's medical record as well as from interviews on the first day after admission and on the day of discharge. They were asked questions about their PA, their general health, and their level of dyspnea before the index eCOPD, 24 hours after being admitted to hospital, and at hospital discharge. Patients discharged from the ED to home were also asked questions related to these variables (their PA, their general health, and their level of dyspnea), in their case, referring to before the index eCOPD, 24 hours after being discharged from the hospital ED, and 1 week after the index ED visit.

For PA level, we focused on walking. Participants were given six response options about their daily activity (walking) before the exacerbation: I just walk from bed to a chair; I am housebound but walk inside the house; I walk a few hundred meters outside; I walk for errands; I regularly walk - walking a walk at least 3 days a week; and I take part in some kind of sport. This PA scale was based on the health, activity, dyspnea, obstruction (HADO) score, ${ }^{15}$ already validated, and has been used in previous studies. This scale showed good correlation with the St George's Respiratory Questionnaire activity subscale. It also shows, in this sample, good correlation with the European Quality of Life-5 Dimensions (EQ-5D; Pearson's $r$ correlation coefficient: 0.42 in admitted patients and 0.49 in those discharged; $P$ for both $<0.0001$ ) and the fatigue scale ( $r$ correlation coefficient: 0.39 and 0.30 , respectively; $P$ for both $<0.0001)$.

At 24 hours after the index ED evaluation, patients were asked about the level of PA they were able to perform at that time, as well as their general health status and dyspnea. Similar response options were given at the time of discharge for patients who had been admitted to the hospital from the ED assessment and after 7 days for those discharged to home from the ED assessment. These were: I am bedridden; I sit down; I walk to the bathroom; I walk in the room; I walk outside the room with limitations; and I can walk outside the room without limitations. Baseline general health status was measured using the EQ-5D questionnaire. ${ }^{16,17}$

To measure the level of dyspnea at baseline we used the Medical Research Council breathlessness scale, which uses a five-category scale that ranges from "I only get breathless with strenuous exercise" to "I am too breathless to leave the house". ${ }^{18}$ The level of dyspnea during the follow-up, that is, 24 hours after the index ED visit and at hospital discharge was measured with a specific question "How do you rate your level of dyspnea today" with a seven-point response scale from very severe to none, similar to the previously validated Borg Dyspnea Scale. ${ }^{19,20}$ 
For all patients, additional variables collected from medical records included pulmonary function as measured by FEV1; hospital admissions for COPD during the previous 12 months; baseline respiratory therapy; and the presence of comorbidities needed to determine the Charlson Comorbidity Index. ${ }^{14}$

Further, for all patients, we reviewed medical records and conducted telephone interviews 2 months after the index ED evaluation to ascertain their vital status and whether they had been readmitted to hospital.

\section{Definitions of outcome measures}

The main outcome variable was change in PA. For patients admitted to the hospital, this was defined as the difference between PA reported at hospital discharge and that reported 24 hours after being admitted to the hospital. For patients discharged to home from the ED, it was defined as the difference between PA reported 1 week after being discharged from the ED and that reported 24 hours after being discharged from the ED to home.

Additional outcome parameters were mortality during the 2 months after the ED index visit; readmission for an eCOPD during these 2 months; and, for patients admitted to the hospital from the index ED visit, length of stay, measured as the day the patient was discharged from hospital minus the day he or she was admitted.

\section{Statistical analysis}

The unit of the analysis was the patient. For patients who had more than one ED visit during the recruitment period, only the first one was considered for the analysis.
Descriptive statistics included frequencies and percentages for categorical variables and means and standard deviation (SD) for continuous variables. Main sociodemographic and clinical characteristics, as well as patient-reported measures at baseline, were compared between patients admitted to hospital from the index ED evaluation and those discharged to home.

Generalized linear models were used in the univariate analysis to determine which variables were related to PA level at discharge among patients admitted to the hospital or 1 week after discharge to home from the ED. Patients who died during this period were excluded from the analysis.

In the multivariate analysis, separate generalized linear models were used for patients admitted to the hospital and those discharged to home from the ED. All variables with a $P$-value lower than 0.20 were included in the multivariate analysis. Variables with $P<0.05$ were included in the final models. The effect of the participating hospital in the final models was also taken into account by means of multilevel models.

All effects were considered to be significant at $P<0.05$. All statistical analyses were performed using SAS for Windows, version 9.2 (SAS Institute Inc., Cary, NC, USA).

\section{Results}

A total of 3,276 eCOPD episodes were identified for the study. The final population included 2,487 patients. The recruitment process is outlined in Figure 1.

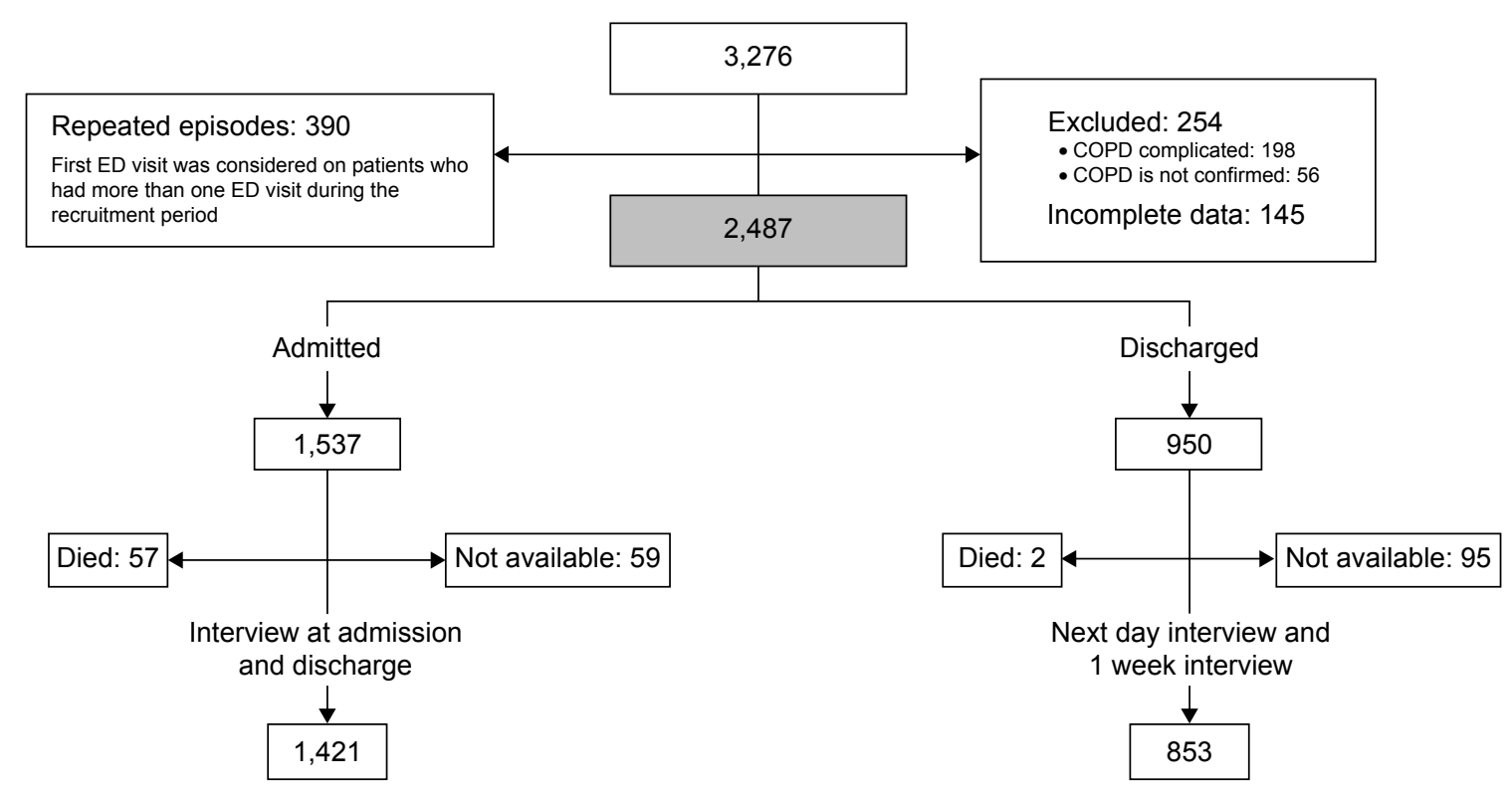

Figure I Flowchart of exclusions and missing cases. Abbreviation: ED, emergency department. 
Both groups of eCOPD patients showed increases in PA during follow-up, that is, at hospital discharge or 1 week after discharge to home from the ED (Figure 2). However, $16 \%$ of the admitted patients did not perform any PA during their hospitalization and $17 \%$ walked only in their hospital room during that time, while among those discharged from the ED the corresponding percentages were $10 \%$ and $9 \%$, respectively (Table 1).

Variables associated with change in PA during hospital admission were: age; baseline FEV1\%; the need for home oxygen therapy; noninvasive mechanical ventilation or continuous positive airway pressure ventilation; number of admissions for an eCOPD during the previous 12 months; Charlson Comorbidity Index score; level of consciousness as measured by the Glasgow Coma Scale score; presence of dyspnea at rest upon ED arrival; length of stay; PA at baseline; and PA in the first 24 hours after admission. Taking the best score in each variable as a reference, all had significant inverse association with change in PA during the hospitalization or the 7 days after being discharged to home from the ED (Table 2).

In multivariate analyses, we evaluated factors associated with the change in PA during hospital admission or an equivalent time ( 1 week) in patients discharged to home from

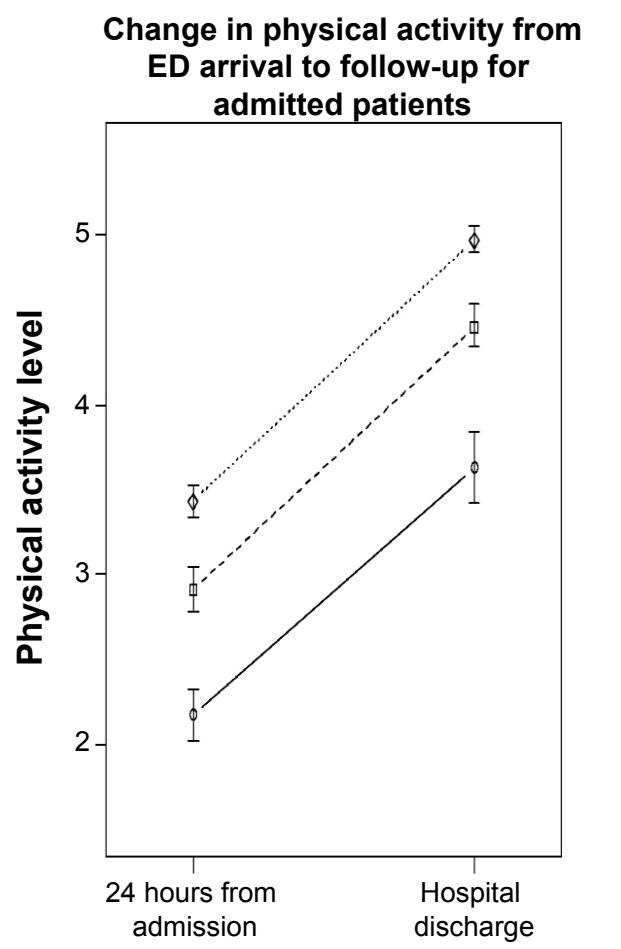

the ED. Among patients admitted to the hospital, changes in PA from admission to discharge (Table 3) were influenced by their previous level of PA: level of PA 24 hours after admission (limited to those patients with the lowest level of PA): age: health-related quality of life (HRQoL) as measured by the EQ-5D: and length of hospital stay: as well as use of inhaled anticholinergic medications (associated with an increase in PA) or corticosteroids (associated with a decrease in PA). Among patients discharged to home from the ED, changes in PA after 1 week (Table 4) were influenced by previous level of PA: level of PA 24 hours after the index ED visit: FEV1\%: and the presence of dyspnea at rest upon ED arrival.

For both groups, patients admitted to the hospital and those discharged to home from the ED, Table 5 shows outcomes including mortality, length of stay, and readmission by PA levels. Among patients admitted to the hospital, the length of hospital stay was associated with the level of PA both at baseline and at 24 hours after admission (falling from 8.9 to 5.3 days with increasing baseline PA and 9.2 to 6.8 with increasing PA at 24 hours, respectively) as was mortality during admission (varying from $15 \%$ to $1 \%$ and $10.5 \%$ to $0 \%$, respectively). Further, readmission rates within 2 months were related to the level of PA at discharge from the

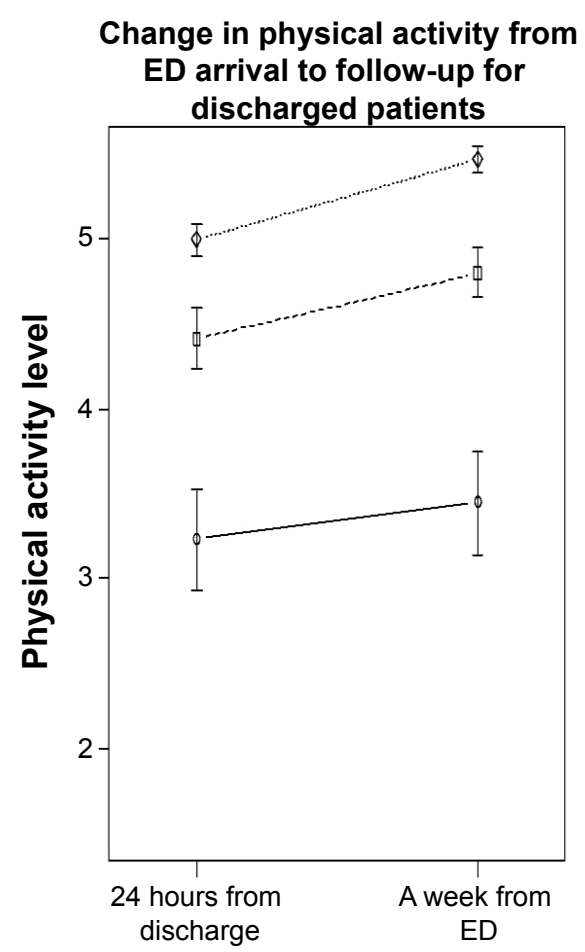

\section{Basal physical activity level $\rightarrow$ Low -8-Moderate $\bullet$. High}

Figure 2 Changes in physical activity from ED arrival to follow-up by basal physical activity level and admission status.

Note: Physical activity levels: I - I am bedridden; 2 - I sit down; 3 - I walk to the bathroom; 4 - I walk in the room; 5 - I walk outside the room with limitations; and 6 - I can walk outside the room without limitations.

Abbreviation: ED, emergency department. 
Table I Descriptive characteristics of the sample by admission status

\begin{tabular}{|c|c|c|c|}
\hline & \multicolumn{2}{|c|}{ Hospital admission } & \multirow[t]{2}{*}{$P$-value } \\
\hline & Yes & No & \\
\hline Age, years & $72.6(9.6)$ & $73.0(9.7)$ & 0.74 \\
\hline Male, \% & I,394 (90.3) & $873(91.8)$ & 0.33 \\
\hline Baseline $\mathrm{FEV}_{1}, \%$ & & & $\leq 0.0001$ \\
\hline$\geq 50$ & $429(30.7)$ & $339(43.1)$ & \\
\hline $30-49$ & $666(47.7)$ & $342(43.5)$ & \\
\hline$<30$ & $302(21.6)$ & $106(13.5)$ & \\
\hline Previous HOT/CPAP/NIMV & $585(38.1)$ & $256(26.9)$ & $\leq 0.0001$ \\
\hline Charlson Comorbidity Index & $2.3 \mathrm{I}(\mathrm{I} .6)$ & $2.10(1.6)$ & 0.002 \\
\hline EQ-5D & $0.60(0.28)$ & $0.64(0.29)$ & 0.003 \\
\hline Previous admission (year before) & $1.0(1.5)$ & $0.56(I . I)$ & $\leq 0.0001$ \\
\hline Living arrangements & & & 0.001 \\
\hline Lives alone & $204(12.6)$ & $81(8.1)$ & \\
\hline Lives in a residence & $38(2.4)$ & $17(1.7)$ & \\
\hline Lives with someone & I,348 (83.6) & $888(89.3)$ & \\
\hline Other & $23(7.4)$ & $9(0.9)$ & \\
\hline Edema (at arrival ED) & $299(21.0)$ & $137(\mid 5.1)$ & $\leq 0.0001$ \\
\hline Dyspnea at rest (at arrival ED) & I,30I (78.5) & $46 \mid(50.7)$ & $\leq 0.0001$ \\
\hline Glasgow Coma Scale < I5 (at ED arrival) & $59(3.8)$ & II (I.I) & 0.0003 \\
\hline Baseline physical activity & & & $\leq 0.0001$ \\
\hline$I=$ from bed to chair only & I0I (7.2) & $56(6.7)$ & \\
\hline $2=$ housebound, but walks inside & $103(7.3)$ & $43(5.1)$ & \\
\hline $3=$ walks a few hundred meters & $363(25.7)$ & $165(19.7)$ & \\
\hline $4=$ walks for errands & $409(29.0)$ & $194(23.2)$ & \\
\hline $5=$ usual walker & $425(30.1)$ & $366(43.8)$ & \\
\hline $6=$ does some sports & $10(0.7)$ & $12(1.4)$ & \\
\hline Physical activity at 24 hours & & & $\leq 0.000 \mathrm{I}$ \\
\hline $\mathrm{I}=$ bedridden & $222(15.6)$ & $31(3.6)$ & \\
\hline $2=$ sits & $234(16.5)$ & $44(5.2)$ & \\
\hline $3=$ walks just to the bathroom & $469(33.0)$ & $69(8.1)$ & \\
\hline $4=$ walks around the room & $246(17.3)$ & $123(14.5)$ & \\
\hline $5=$ walks outside of the room with limitations & $212(14.9)$ & $38 \mathrm{I}(45.0)$ & \\
\hline $6=$ walks outside of the room without limitations & $37(2.6)$ & $199(23.5)$ & \\
\hline Physical activity at I week/discharge & & & $\leq 0.0001$ \\
\hline $\mathrm{I}=$ bedridden & $35(2.6)$ & $24(2.9)$ & \\
\hline $2=$ sits & $60(4.5)$ & $24(2.9)$ & \\
\hline $3=$ walks just to the bathroom & II4 (8.5) & $32(3.8)$ & \\
\hline $4=$ walks around the room & $227(17.0)$ & $74(9.0)$ & \\
\hline $5=$ walks outside of the room with limitation & $628(46.9)$ & $342(4 \mid .5)$ & \\
\hline $6=$ walks outside of the room without limitation & $274(20.4)$ & $328(39.8)$ & \\
\hline Length of stay (admitted) & $7.75(6.2)$ & $\mathrm{N} / \mathrm{A}$ & \\
\hline Death & $56(3.6)$ & $3(0.3)$ & $\leq 0.000 \mathrm{I}$ \\
\hline
\end{tabular}

Notes: Summary variables are presented as mean \pm standard deviation for quantitative variables, and frequency for discrete variables.

Abbreviations: $\mathrm{FEV}_{1}$, forced expiratory volume in I second; HOT, home oxygen therapy; CPAP, continuous positive airways pressure; N/A, not applicable; NIMV, noninvasive mechanical ventilation; EQ-5D, European Quality of Life-5 Dimensions; ED, emergency department.

hospital, or 1 week after the index visit in patients discharged (varying from $40 \%$ to $15 \%$ and $41 \%$ to $11 \%$, respectively, with increasing levels of PA).

\section{Discussion}

The results of this study indicate that the level of PA is quite variable during an eCOPD that requires an ED visit, as are the variables associated with the change in PA during the exacerbation, and these differ between patients admitted to hospital and those discharged to home. PA levels at baseline, 24 hours after hospital admission, and at the time of hospital discharge or 7 days after the index ED assessment are associated with length of stay, readmission, and mortality during admission. 
Table 2 Univariate analysis of predictors of change of physical activity at hospital discharge or 7 days after visiting the ED

\begin{tabular}{|c|c|c|c|c|}
\hline & \multicolumn{4}{|c|}{ Hospital admission } \\
\hline & \multicolumn{2}{|l|}{ Yes } & \multicolumn{2}{|l|}{ No } \\
\hline & $\beta$ estimate & $P$-value & $\beta$ estimate & $P$-value \\
\hline Age & -0.01 & $\leq 0.000 \mathrm{I}$ & -0.02 & $\leq 0.0001$ \\
\hline \multicolumn{5}{|l|}{ Sex } \\
\hline Men & -0.08 & 0.48 & -0.001 & 0.99 \\
\hline Women & - & - & - & - \\
\hline \multicolumn{5}{|l|}{ Baseline $\mathrm{FEV}_{1}, \%$} \\
\hline$\geq 50$ & - & - & - & - \\
\hline $30-49$ & -0.23 & 0.003 & -0.38 & $\leq 0.000 \mathrm{I}$ \\
\hline$<30$ & -0.38 & $\leq 0.0001$ & -0.75 & $\leq 0.000 \mathrm{I}$ \\
\hline \multicolumn{5}{|l|}{ Previous HOT/CPAP/NIMV } \\
\hline No & - & - & - & - \\
\hline Yes & -0.48 & $\leq 0.000 \mathrm{I}$ & -0.50 & $\leq 0.0001$ \\
\hline Charlson Comorbidity Index & -0.08 & $\leq 0.0001$ & -0.06 & 0.01 \\
\hline Previous admission (year before) & -0.08 & $\leq 0.0001$ & -0.08 & 0.05 \\
\hline \multicolumn{5}{|l|}{ Living arrangements } \\
\hline Lives alone & 0.25 & 0.01 & 0.28 & 0.05 \\
\hline Lives in a residence & -0.59 & 0.002 & -1.1 & 0.0007 \\
\hline Other & -0.43 & 0.14 & -1.03 & 0.009 \\
\hline Lives with someone & - & - & - & - \\
\hline \multicolumn{5}{|l|}{ Edema (at arrival ED) } \\
\hline No & - & - & - & - \\
\hline Yes & -0.36 & 0.001 & -0.13 & 0.10 \\
\hline \multicolumn{5}{|l|}{ Dyspnea at rest (at arrival ED) } \\
\hline No & - & - & - & - \\
\hline Yes & -0.28 & 0.0003 & -0.37 & $\leq 0.0001$ \\
\hline \multicolumn{5}{|l|}{ Glasgow Coma Scale $<15$ (at arrival ED) } \\
\hline No & - & - & - & - \\
\hline Yes & -0.65 & 0.0006 & -1.24 & 0.0008 \\
\hline \multicolumn{5}{|l|}{ Baseline physical activity } \\
\hline $\mathrm{I}=$ from bed to chair life only & -2.00 & $\leq 0.0001$ & -2.49 & $\leq 0.0001$ \\
\hline $2=$ housebound, but could walk inside & -1.37 & 0.0001 & -1.36 & $\leq 0.0001$ \\
\hline $3=$ walks a few hundred meters & -0.85 & 0.01 & -0.66 & 0.02 \\
\hline $4=$ walks for errands & -0.51 & 0.14 & -0.22 & 0.46 \\
\hline $5=$ usually walker & -0.24 & 0.47 & -0.002 & 0.99 \\
\hline $6=$ does some sports & - & - & - & - \\
\hline \multicolumn{5}{|l|}{ Physical activity at 24 hours after ED arrival } \\
\hline $\mathrm{I}=$ bedridden & -1.59 & $\leq 0.000 \mathrm{I}$ & -2.80 & $\leq 0.0001$ \\
\hline $2=$ sits & -1.22 & $\leq 0.0001$ & -2.21 & $\leq 0.0001$ \\
\hline $3=$ walks just to the bathroom & -0.79 & $\leq 0.0001$ & -1.26 & $\leq 0.0001$ \\
\hline $4=$ walks around the room & -0.56 & 0.005 & -0.88 & $\leq 0.0001$ \\
\hline $5=$ walks outside of the room with limitations & -0.25 & 0.22 & -0.49 & $\leq 0.0001$ \\
\hline $6=$ walks outside of the room without limitations & - & - & - & - \\
\hline Length of stay & -0.03 & $\leq 0.0001$ & - & - \\
\hline
\end{tabular}

Note: Reference categories for each variable were the best situation category for each variable.

Abbreviations: $\mathrm{FEV}_{1}$, forced expiratory volume in I second; HOT, home oxygen therapy; CPAP, continuous positive airways pressure; NIMV, noninvasive mechanical ventilation; ED, emergency department.

Hospitalization has previously been associated with decreases in PA. Fisher et al found that older people hospitalized for an acute medical illness performed little PA, spending only $4.1 \%$ of their time walking. ${ }^{21}$ In 17 hospitalized patients with severe COPD who were fitted with accelerometers, Pitta et al found that PA was significantly lower than the PA level of patients with stable COPD. ${ }^{7}$ In contrast, our study showed that COPD patients have a wide range of PA during a hospital admission for an eCOPD. In fact, in the first 24 hours after admission, $17.5 \%$ of patients in our study walked, at least, in their hospital rooms. Apart from the use of different measurement tools, these differences 
Table 3 Change in PA level in admitted patients: multivariate analysis

\begin{tabular}{|c|c|c|}
\hline & $\beta$ estimate & $P$-value \\
\hline Intercept & 5.63 & $<0.0001$ \\
\hline \multicolumn{3}{|l|}{ Baseline physical activity } \\
\hline From "bed to chair life" or housebound, but walks inside & -0.61 & $<0.0001$ \\
\hline Walks a few hundred meters & -0.16 & 0.026 \\
\hline Walks for errands or usually walker or does some sports & - & - \\
\hline \multicolumn{3}{|l|}{ Physical activity at 24 hours } \\
\hline I= bedridden & -0.64 & 0.001 \\
\hline $2=$ sits & -0.54 & 0.005 \\
\hline $3=$ walks just to the bathroom & -0.36 & 0.05 \\
\hline $4=$ walks around the room & -0.30 & 0.11 \\
\hline $5=$ walks outside of the room with limitation & -0.12 & 0.54 \\
\hline $6=$ walks outside of the room without limitation & - & - \\
\hline Age & -0.012 & $<0.0001$ \\
\hline EQ-5D & 0.95 & $<0.0001$ \\
\hline \multicolumn{3}{|l|}{ Home status } \\
\hline Lives alone & 0.21 & 0.015 \\
\hline Lives in a residence & -0.14 & 0.43 \\
\hline Other & -0.18 & 0.49 \\
\hline Lives with someone & - & - \\
\hline \multicolumn{3}{|l|}{ Medication } \\
\hline Anticholinergic (used) & 0.16 & 0.03 \\
\hline Anticholinergic (not used) & - & - \\
\hline Corticosteroids (systemic) used & -0.27 & 0.0005 \\
\hline Corticosteroids (systemic) not used & - & - \\
\hline Length of hospital stay & -0.012 & 0.03 \\
\hline
\end{tabular}

Table 4 Change in PA level in ED discharged patients: multivariate analysis

\begin{tabular}{|c|c|c|}
\hline & $\beta$ estimate & $P$-value \\
\hline Intercept & 5.95 & $<0.000$ I \\
\hline \multicolumn{3}{|l|}{ Baseline PA } \\
\hline From bed to chair life only or housebound, but could walk inside & -1.17 & $<0.000$ I \\
\hline Walks a few hundred meters & -0.32 & 0.0005 \\
\hline Walks for errands or usually walker or does some sports & - & - \\
\hline \multicolumn{3}{|l|}{ Physical activity at 24 hours } \\
\hline $\mathrm{I}=$ bedridden & -2.04 & $\leq 0.000 \mathrm{I}$ \\
\hline $2=$ sits & -1.52 & $\leq 0.000 \mathrm{I}$ \\
\hline $3=$ walks just to the bathroom & -0.94 & $\leq 0.000 \mathrm{I}$ \\
\hline $4=$ walks around the room & -0.68 & $\leq 0.000 \mathrm{I}$ \\
\hline $5=$ walks outside of the room with limitation & -0.41 & $\leq 0.000 \mathrm{I}$ \\
\hline $6=$ walks outside of the room without limitation & - & - \\
\hline \multicolumn{3}{|l|}{$\mathrm{FEV}_{1}, \%$} \\
\hline$<30$ & -0.23 & 0.04 \\
\hline $30-49$ & -0.19 & 0.01 \\
\hline$\geq 50$ & - & - \\
\hline \multicolumn{3}{|l|}{ Dyspnea at rest at ED arrival } \\
\hline Yes & -0.16 & 0.03 \\
\hline No & - & - \\
\hline
\end{tabular}

Notes: Model $R$-square: 0.43 , reference categories for each variable were the best case category for each variable; baseline physical activity categories were reduced to three levels for this model.

Abbreviations: ED, emergency department; PA, physical activity; $\mathrm{FEV}_{1}$, forced expiratory volume in I second. 
Table 5 Outcomes by physical activity levels in patients admitted and discharged from ED

\begin{tabular}{|c|c|c|c|c|c|c|c|}
\hline \multirow[t]{2}{*}{ Outcomes } & \multicolumn{6}{|c|}{ Physical activity level } & \multirow[t]{2}{*}{$P$-value } \\
\hline & $\mathbf{I}$ & 2 & 3 & 4 & 5 & 6 & \\
\hline \multicolumn{8}{|l|}{ Hospital admitted patients } \\
\hline \multicolumn{8}{|l|}{ Baseline physical activity } \\
\hline Length of stay & $8.9(5.1)$ & $10.2(7.7)$ & $8.8(6.6)$ & $7.5(5.0)$ & $6.1(3.8)$ & $5.3(4.1)$ & $\leq 0.000 \mathrm{I}$ \\
\hline Death during admission & $19(15.45)$ & II (9.17) & $9(2.12)$ & $3(0.67)$ & $\mathrm{I}(\mathrm{I} .25)$ & $\mathrm{I}(0.25)$ & $\leq 0.0001$ \\
\hline \multicolumn{8}{|l|}{ Physical activity at 24 hours } \\
\hline Length of stay & $9.2(6.3)$ & $8.9(5.8)$ & $7.6(5.7)$ & $7.2(4.7)$ & $5.8(3.9)$ & $6.8(5.6)$ & $\leq 0.0001$ \\
\hline Death during admission & $27(10.55)$ & $7(2.67)$ & $7(1.31)$ & $3(1.06)$ & $0(0)$ & $0(0)$ & $\leq 0.000 \mathrm{I}$ \\
\hline \multicolumn{8}{|l|}{ Physical activity at discharge } \\
\hline Readmissions until 2 months & $16(40)$ & $17(25.37)$ & $38(29.92)$ & 65 (25.39) & $142(20.40)$ & $4 \mid(\mid 5.65)$ & 0.001 \\
\hline \multicolumn{8}{|l|}{ ED discharged patients } \\
\hline \multicolumn{8}{|l|}{ Physical activity at I week } \\
\hline Readmissions until 2 months & II (40.74) & $7(34.14)$ & $7(19.44)$ & $22(26.5 \mathrm{I})$ & $53(12.68)$ & $33(10.61)$ & $\leq 0.000 \mathrm{I}$ \\
\hline
\end{tabular}

Notes: Summary variables are presented as mean \pm standard deviation for quantitative variables, and frequencies and percentage for discrete variables. Baseline physical activity: I, from bed to chair only; 2, "housebound but I walk inside"; 3, "I walk a few hundred meters outside"; 4, "I walk for errands"; 5, "I regularly walk - walk an itinerary at least 3 days a week"; 6 , "I do some kind of sports". Physical activity at 24 hours, physical activity at discharge, physical activity at I week after being discharged from ED: I, "I am bedridden"; 2, "I sit"; 3, "I walk just to the bathroom"; 4, "I walk in the room"; 5, "I walk outside the room with limitation"; 6, "I can walk outside room without limitations”.

Abbreviation: ED, emergency department.

could be explained by the larger size of our cohort and the range of COPD severity among the participants.

Little information is available about PA among patients experiencing an eCOPD who are not admitted to hospital. As might be expected, in our cohort these patients had a higher level of PA in the first 24 hours after the index ED visit than those who were admitted to the hospital. Only $17 \%$ of patients discharged to home from the ED reported minimal PA at 24 hours (categories 1-3) compared to $65 \%$ of admitted patients.

Donaldson et al showed that COPD patients spent only 1.5 days indoors immediately after an exacerbation - not necessarily a severe one - which implies a quick return to normality. ${ }^{9}$ In contrast, Pitta et al found that several patients failed to achieve the PA level of stable COPD patients 1 month after their hospital discharge. ${ }^{10}$ Our data for nonadmitted eCOPD patients were generally in agreement with a relatively fast recovery.

Some factors associated with the change in PA during hospital admission are related to the general clinical condition of the patient, such as older age, poorer HRQoL, living alone, and reporting the lowest level of PA at baseline (confined to bed or a chair). Other associated variables were PA during the first 24 hours after admission, length of stay, and use of anticholinergic or systemic corticosteroids to treat the eCOPD.

Interestingly, among patients admitted to the hospital, factors related to the severity of COPD, such as FEV1\%, level of dyspnea when the patient was stable, comorbidities, and hospitalizations for eCOPD in the previous year played no role in the change of PA during the admission. Further, even variables related to the ED visit (reflecting the severity of the exacerbation), such as level of respiratory failure, also had no relationship with the change in PA. These findings are in accordance with the results of Fisher et al who found no association between the number of steps taken by patients during admission and the severity of the illness. ${ }^{21}$

Among patients admitted to the hospital, the lower the PA level during the first 24 hours after admission, the smaller the change in PA at hospital discharge. This finding suggests that early activity might improve later PA and perhaps other outcomes among patients hospitalized for an eCOPD.

In our study, HRQoL was related to the change in PA during hospitalization. Among stable COPD patients, HRQoL has previously been identified as a predictor of PA. ${ }^{22} \mathrm{An}$ association between changes in PA and changes in HRQoL has been also observed, ${ }^{23}$ although such an association has not been demonstrated during an eCOPD.

Among patients discharged to home from the ED, the only variables of the eCOPD episode that were associated with changes in PA were baseline level of PA, FEV1\%, and dyspnea at rest upon arrival at the ED.

Surprisingly, the use of anticholinergic medication during admission was associated with a positive change in PA. This could reflect appropriate treatment of a severe eCOPD that improves hyperinflation and makes PA easier. ${ }^{24}$ In contrast, the use of systemic corticosteroids was associated with a decline in PA, which could be related to deterioration of muscle function. ${ }^{10}$ 
Summarizing, in both groups of our sample, we identified important factors beyond to the two common predictive variables, namely, PA at baseline and 24 hours. Among those discharged from the ED, these predictors were just baseline COPD obstructive severity level and dyspnea status at ED arrival; while for those admitted several parameters related to general health status of the patient (age, EQ-5D, and living arrangements) and, importantly, to the index admission (length of stay and medication used) make a difference to the prediction of changes. These findings indicate that variables besides COPD status, including some treatments, play an important role.

Several studies have suggested that PA may be therapeutic during hospitalization. Among patients hospitalized for an acute medical condition, those who had shorter lengths of stay took more steps on the first complete day in hospital. ${ }^{21}$ In another study by Fisher et al patients hospitalized for an acute medical illness who increased their walking by at least 600 steps from the first to the second 24-hour day were discharged 1.7 days earlier than those who did not. ${ }^{6}$ Shadmi and Zisberg observed a 1.5-day shorter length of stay in patients who were mobile outside their hospital rooms compared with those who were only mobile inside their rooms. ${ }^{25}$

Low-grade systemic inflammation is part of the COPD disease process. Skeletal muscle wasting has been related to the use of systemic corticosteroids and immobilization, and has been associated with functional deterioration during hospitalization in eCOPD patients. ${ }^{10} \mathrm{PA}$ would increase the circulating level of anti-inflammatory cytokines such as interleukin (IL)-6, IL-1 ra, IL-10, and soluble tumor necrosis factor receptors, ${ }^{26}$ which could help restore equilibrium in this inflammatory state ${ }^{27}$ (although it is not known which agents mediate this effect). A rehabilitation program undertaken during admission for eCOPD has been shown to prevent muscle deterioration. ${ }^{28} \mathrm{We}$ speculate that early mobilization would work as a kind of rehabilitation, with similar benefits to a structured program.

The multicenter design and the large number of patients included in the two arms of the study - those admitted and those directly discharged from the ED - are the main strength of this study.

Limitations of our study should also be noted. We did not objectively measure PA with accelerometers, as has been done in smaller studies. Using these devices in a large, multicenter study such as ours would have been extremely complicated. Prospective studies are likely to have missing data, this being inherent to the design. Our study is no exception, although we were able to include a high proportion of patients at follow-up and collect complete information for the variables considered in the analysis.

In summary, we found that among patients evaluated in an ED for an eCOPD the level of PA is quite variable. Immediately following the $\mathrm{COPD}$, variables related to the exacerbation and those reflecting the general clinical condition of the patient influence changes in the level of PA, especially among those admitted to hospital from the ED. Future studies should confirm these results using objective PA measurements. Maximizing bronchodilator treatment and beginning PA at a very early stage following an eCOPD could improve outcomes in these patients.

\section{Acknowledgments}

We are grateful for the support of the 16 participating hospitals (Hospital Costa del Sol, Hospital Valme, Hospital de Motril, Corporació Sanitaria Parc Taulí, Hospital del Mar, Hospital Universitario de La Princesa, Hospital Universitario Gregorio Marañón, Hospital Universitario La Paz, Hospital de Móstoles, Hospital Marqués de Valdecilla, Hospital Santa Marina, Hospital San Eloy, Hospital Galdakao-Usansolo, Hospital Txagorritxu, Complejo Hospitalario Donostia, and Hospital Cruces), as well as the ED physicians, other clinicians, and staff members of the various services, research units, quality units, and medical records sections of these hospitals. We also gratefully acknowledge the patients who participated in the study.

This work was supported in part by grants from the Fondo de Investigación Sanitaria (PI 06\1010, PI06\1017, PI06\714, PI06\0326, PI06\0664); Department of Health of the Basque Country, Department of Education, Universities and Research of the Basque Government (UE09/62); the Research Committee of the Hospital Galdakao; and the thematic networks - Red IRYSS (Investigacion en Resultados y Servicios Sanitarios (G03/220) - of the Instituto de Salud Carlos III.

The authors also acknowledge the editorial assistance provided by Patrick Skerrett.

The IRYSS-COPD group included the following coinvestigators: Dr Jesús Martínez-Tapias (Hospital Virgen de las Nieves, Granada); Alba Ruiz (Hospital de Motril, Granada); Dr Eduardo Briones (Unidad de Epidemiología. Distrito Sanitario Sevilla); Dra Silvia Vidal (Unidad de Investigación, Hospital Costa del Sol, Marbella); Dr Emilio Perea-Milla, Francisco Rivas (Servicio de Epidemiología, Hospital Costa del Sol, Málaga - REDISSEC); Dr Maximino Redondo (Servicio de Laboratorio, Hospital Costa del Sol, Málaga-REDISSEC); Javier Rodríguez Ruiz (Responsable de Enfermería del Área de Urgencias, Hospital Costa del 
Sol, Málaga); Dra Marisa Baré (Epidemiología y Evaluación, Corporació Sanitaria Parc Taulí-CSPT, Sabadell REDISSEC), Dr Manel Lujan, Dra Concepción Montón (Servicio de Neumología, CSPT/REDISSEC); Dra Amalia Moreno, Dra Josune Ormaza, Dr Javier Pomares (Servicio de Neumología, CSPT); Dr Juli Font (Medicina, Servicio de Urgencias; CSPT), Dra Cristina Estirado, Dr Joaquín Gea (Servicio de Neumología, Hospital del Mar/CIBERES, Barcelona); Dra Elena Andradas (subdirectora de Promoción de la Salud y Epidemiología del Ministerio de Sanidad, Servicios Sociales e Igualdad), Dr Juan Antonio Blasco (Unidad de Evaluación de Tecnologías Sanitarias, Agencia Laín Entralgo, Madrid), Dra Nerea Fernández de Larrea (Subdirección General de Tecnología e Innovación Sanitarias. Consejería de Sanidad de la Comunidad de Madrid/REDISSEC); Rosa Girón (Hospital de La Princesa, Madrid), María del Puerto Cano Aguirre (Hospital de Torrejón, Madrid); Dr Jose Luis Lobo (Servicio de Neumología, Hospital Txagorritxu, Araba); Dra Esther Pulido (Servicio de Urgencias, Hospital Galdakao-Usansolo, Bizkaia); Dr Mikel Sánchez (Servicio de Urgencias, Hospital Galdakao-Usansolo); Dr Luis Alberto Ruiz (Servicio de Respiratorio, Hospital de Cruces, Bizkaia); Dra Ane Miren Gastaminza (Hospital San Eloy, Bizkaia); Dra Eva Tabernero (Servicio de Neumología, Hospital de Santa Marina), Carmen Haro (Servicio de Urgencias, Hospital de Santa Marina, Bizkaia); Dr Ramon Agüero (Servicio de Neumología, Hospital Marqués de Valdecilla, Santander); Dr Gabriel Gutiérrez (Servicio de Urgencias, Hospital Cruces, Bizkaia); Dra Belén Elizalde (Dirección Territorial de Gipuzkoa); Dr Felipe Aizpuru (Unidad de Investigación, Hospital Txagorritxu, Álava/ REDISSEC); Dra Inmaculada Arostegui, Irantzu Barrio (Departamento de Matemática Aplicada, Estadística e Investigación Operativa, UPV/EHU- REDISSEC); Amaia Bilbao (Hospital Universitario Basurto/REDISSEC); Dr Cristóbal Esteban (Servicio de Neumología, Hospital GaldakaoUsansolo, Bizkaia/REDISSEC); Dra Nerea González, Susana Garcia, Iratxe Lafuente, Urko Aguirre; Miren Orive, Ane Anton, and Dr Jose M Quintana (Unidad de Investigación, Hospital Galdakao-Usansolo, Bizkaia/REDISSEC).

\section{Disclosure}

The authors report no conflicts of interest in this work.

\section{References}

1. Buurman BM, van Munster BC, Korevaar JC, de Haan RJ, de Rooij SE. Variability in measuring (instrumental) activities of daily living functioning and functional decline in hospitalized older medical patients: a systematic review. J Clin Epidemiol. 2011;64:619-627.

2. McCusker J, Kakuma R, Abrahamowicz M. Predictors of functional decline in hospitalized elderly patients: a systematic review. J Gerontol A Biol Sci Med Sci. 2002;57:M569-M577.
3. Covinsky KE, Palmer RM, Counsell SR, Pine ZM, Walter LC, Chren MM. Functional status before hospitalization in acutely ill older adults: validity and clinical importance of retrospective reports. J Am Geriatr Soc. 2000;48:164-169.

4. Brown CJ, Redden DT, Flood KL, Allman RM. The underrecognized epidemic of low mobility during hospitalization of older adults. $J \mathrm{Am}$ Geriatr Soc. 2009;57:1660-1665.

5. Brown CJ, Friedkin RJ, Inouye SK. Prevalence and outcomes of low mobility in hospitalized older patients. J Am Geriatr Soc. 2004;52: 1263-1270.

6. Fisher SR, Kuo YF, Graham JE, Ottenbacher KJ, Ostir GV. Early ambulation and length of stay in older adults hospitalized for acute illness. Arch Intern Med. 2010;170:1942-1943.

7. Pitta F, Troosters T, Spruit MA, Probst VS, Decramer M, Gosselink R. Characteristics of physical activities in daily life in chronic obstructive pulmonary disease. Am J Respir Crit Care Med. 2005;171: 972-977.

8. Esteban C, Arostegui I, Aburto M, et al. Influence of changes in physical activity on frequency of hospitalization in chronic obstructive pulmonary disease. Respirology. 2014;19:330-338.

9. Donaldson GC, Wilkinson TM, Hurst JR, Perera WR, Wedzicha JA. Exacerbations and time spent outdoors in chronic obstructive pulmonary disease. Am J Respir Crit Care Med. 2005;171:446-452.

10. Pitta F, Troosters T, Probst VS, Spruit MA, Decramer M, Gosselink R. Physical activity and hospitalization for exacerbation of COPD. Chest. 2006;129:536-544.

11. Quintana JM, Esteban C, Barrio I, et al. The IRYSS-COPD appropriateness study: objectives, methodology, and description of the prospective cohort. BMC Health Serv Res. 2011;11:322.

12. The Global Strategy for the Diagnosis, Management and Prevention of Chronic Obstructive Pulmonary Disease. Global Initiative for Chronic Obstructive Lung Disease (GOLD); 2011. Available from: http://www. goldcopd.org/. Accessed Jan 21, 2011.

13. Teasdale G, Jennett B. Assessment of coma and impaired consciousness. A practical scale. Lancet. 1974;2:81-84.

14. Charlson ME, Pompei P, Ales KL, MacKenzie CR. A new method of classifying prognostic comorbidity in longitudinal studies: development and validation. J Chronic Dis. 1987;40:373-383.

15. Esteban C, Quintana JM, Aburto M, Moraza J, Capelastegui A. A simple score for assessing stable chronic obstructive pulmonary disease. QJM. 2006;99:751-759.

16. Brooks R. EuroQol: the current state of play. Health Policy. 1996;37: 53-72.

17. Badia X, Roset M, Montserrat S, Herdman M, Segura A. [The Spanish version of EuroQol: a description and its applications. European Quality of Life scale]. Med Clin (Barc). 1999;112 Suppl 1:79-85.

18. Fletcher CM, Elmes PC, Fairbairn AS, Wood CH. The significance of respiratory symptoms and the diagnosis of chronic bronchitis in a working population. Br Med J. 1959;2:257-266.

19. Hareendran A, Leidy NK, Monz BU, Winnette R, Becker K, Mahler DA. Proposing a standardized method for evaluating patient report of the intensity of dyspnea during exercise testing in COPD. Int $J$ Chron Obstruct Pulmon Dis. 2012;7:345-355.

20. Marin JM, Carrizo SJ, Gascon M, Sanchez A, Gallego B, Celli BR. Inspiratory capacity, dynamic hyperinflation, breathlessness, and exercise performance during the 6-minute-walk test in chronic obstructive pulmonary disease. Am J Respir Crit Care Med. 2001;163: $1395-1399$.

21. Fisher SR, Goodwin JS, Protas EJ, et al. Ambulatory activity of older adults hospitalized with acute medical illness. J Am Geriatr Soc. 2011;59: 91-95.

22. García-Aymerich J, Félez MA, Escarrabill J, et al. Physical activity and its determinants in severe chronic obstructive pulmonary disease. Med Sci Sports Exerc. 2004;36:1667-1673.

23. Esteban C, Quintana JM, Aburto M, et al. Impact of changes in physical activity on health-related quality of life among patients with COPD. Eur Respir J. 2010;36:292-300. 
24. O'Donnell DE, Laveneziana P. The clinical importance of dynamic lung hyperinflation in COPD. COPD. 2006;3:219-232.

25. Shadmi E, Zisberg A. In-hospital mobility and length of stay. Arch Intern Med. 2011;171:1298.

26. Steensberg A, Fischer CP, Keller C, Møller K, Pedersen BK. IL-6 enhances plasma IL-1ra, IL-10, and cortisol in humans. Am J Physiol Endocrinol Metab. 2003;285:E433-E437.
27. Brandt C, Pedersen BK. The role of exercise-induced myokines in muscle homeostasis and the defense against chronic diseases. J Biomed Biotechnol. 2010;2010:520258. doi:10.1155/2010/520258.

28. Troosters T, Probst VS, Crul T, et al. Resistance training prevents deterioration in quadriceps muscle function during acute exacerbations of chronic obstructive pulmonary disease. Am J Respir Crit Care Med. 2010;181:1072-1077.

International Journal of COPD

\section{Publish your work in this journal}

The International Journal of COPD is an international, peer-reviewed journal of therapeutics and pharmacology focusing on concise rapid reporting of clinical studies and reviews in COPD. Special focus is given to the pathophysiological processes underlying the disease, intervention programs, patient focused education, and self management protocols.

\section{Dovepress}

This journal is indexed on PubMed Central, MedLine and CAS. The manuscript management system is completely online and includes a very quick and fair peer-review system, which is all easy to use. Visit $\mathrm{http}: / / \mathrm{www}$.dovepress.com/testimonials.php to read real quotes from published authors.

Submit your manuscript here: http://www.dovepress.com/international-journal-of-chronic-obstructive-pulmonary-disease-journal 